\title{
Design and Materials Characterization Issues in Microstructure Technology (MST)
}

\author{
Ajay Kumar Rakesh \\ Department of Physics,M.M.H. College,Ghaziabad (UP),India
}

\begin{abstract}
Microstructure technology (MST) has become an important area of technology, building on the success of the microelectronics industry over the past 50 years. MSTT combines mechanical and electrical function in devices at very small scales. Examples include pressure sensors, accelerometers, gyroscopes and optical devices, as well as chemical, biomedical and fuidic applications.The status of Microstructure technology is reviewed with particular emphasis on materials issues therein. The materials issues in MST are divided into three categories, the MST material set, microfabrication processes, and material characterization and design. Of these areas, here particular emphasis is given on characterization and design in MST. A discussion on the future of MST specially emphasis on characterization is given.
\end{abstract}

Keywords: MST,MEMS,Microstructure technology; Scaling; Microfabrication; Material selection; Mechanical Properties.

\section{INTRODUCTION}

The past decade has seen the rapid growth of microelectromechanical systems (MEMS) as an important area of microstructure technology, growth which is expected to continue well into the next century. The basic premise behind the concept of MST is that the effciencies of high volume production and low unit cost achieved by the microelectronics industry over the past 50 years can be translated to devices in which mechanical and electrical components are integrated within a single silicon chip (or equivalent structure). In addition to the potential economic benefits, unique capabilities can be achieved by such integration to realize devices at very small scales such as sensors, actuators, power producing devices, chemical reactors and bio-medical devices. Furthermore, the ability to integrate the mechanical (or biological or chemical)function with the electronics required for controland power conditioning in a single device allows for consideration of concepts such as the highly distributed networks required for health monitoring of large structures and systems or for distributed power and chemical production schemes. The success of MEMS and MST as a key technology in the twenty first century depends in no small part on the solution of materials issues associated with the design and fabrication of complex MST devices. The small scales of MEMS offers the opportunity to exploit materials which would not normally be available for large scale devices as well as taking advantage of scale dependent properties, particularly yield and fracture strength . MEMS also offer the opportunity to materials scientists and engineers to be able to characterize materials in ways that have not hitherto been possible. In this article the current status of MEMS is reviewed with a particular emphasis on the role of materials, as well as some of the opportunities for MEMS to contributeto the wider field of materials science and engineering.Before focusing on materials issues in MEMS it is important to make some statements regarding the scope of this article. First, small size and use of some microfabrication processes in its creation does not automatically qualify a device to be defined as a MEMS. The two key attributes in the defnition used here are that the microfabrication processes used to create the device should be scaleable in order to realize a low unit cost of production and that there is some level of integration between electronic and non-electronic function. The effect of these working defnitions is to confine the discussion to devices and processes that utilize or build from the experience established with microelectronics,however it does permit discussion of devices other than sensors and actuators which have hitherto been the focus of MEMS development. The infuence of this restriction will be assessed in the discussion section toward the end of the article.The second key point is that, as their acronym suggests, MEMS are systems. Thus a description of challenges and the development of solutions for MEMS must be presented in the context of the overall system, as opposed to solutions that only address one facet of performance. The introduction of a new material to address a particular aspect of the mechanical performance of a device is worthless unless a fabrication route exists which is compatible with the other materials and structures within the device, and that the new material provides the appropriate functionality in the context of system performance. A corollary of this observation is that many of the materials issues are not unique to MEMS, and materials solutions would be likely to find application in other areas. In this article particular attention is given to materials issues which are of principal importance to MEMS, whereas issues that are common to other applications, such as microelectronic devices or packages, are only mentioned in passing. The realization that materials technologies are 
enabling or limiting for some MEMS concepts has spurred a growing interest in MEMS within the materials community. Several symposia and workshops on MEMS and materials have been held over the past years and the proceedings of these meetings have helped frame the issues discussed in this paper. Interested readers are also referred to several excellent broader references/reviews of MEMS technology and microfabrication.

\section{DESIGN ISSUES AND MATERIALS CHARACTERIZATION}

A main reason for the consistant technical progress and economic growth of the microelectronics industry is the speed and confidence with which complex products can be designed without the need for extensive prototyping. Design in microelectronicdevices is mainly enabled by the reliability of the simulation tools available and the extremely well characterized electronic properties of the materials being used and the processes with which the products are created. For MEMS to achieve their promise of low unit cost and large volume production it is important that similar design procedures be developed. Several simulation tools have been developed to address this need and various packages are available commercially and are particularly used in the design of highly integrated MEMS devices. The development of standardized test methods and material property data bases has lagged behind that of the design and simulation tools, limiting their utility. As early as 1986 the need to develop such a capability was recognized, but it is only recently that wide scale activity has been directed in this area. The issue here is that microfabricated materials have properties that are highly dependent on the fabrication route used to create them and the scale of the structures that they constitute. The mechanical properties at the microscale can vary considerably from those measured on bulk samples of material at the macroscale. Even properties such as density and elastic modulus which are not inherently scale-dependent can be altered from bulk values in deposited layers by the creation of non-equilibrium microstructures, dissolved gases from vapor deposition and the influence of the substrate.In order to fully realize the potential for accurate and rapid simulation tools for the design of MEMS, models are required which link the material property achieved, to the fabrication route and ma-terial used. The first step towards this is to develop standard test methods with which to characterize the mechanical properties of microfabricated material produced by the same processes and at the same scales as the intended application. This will enable the creation of validated material property and process data bases and correlations between processing route and properties, to permit simulation based design. The following sections illustrate where progress in this direction has been made.

\subsection{Elastic properties-}

Perhaps the most mature area of material testing is the measurement of elastic properties of micro-fabricated materials using simple test structures.Cantilever beams and diaphragms which are loadedelectrostically, mechanically by nanoprobes, or by fluid pressure, with deflections measured by means of capacitance or optical sensors have also been extensively used. Resonant structures have also been utilized for this purpose and offer the potential for extremely accurate measurements. These methods have allowed reproducible evaluation of the Young's moduli of deposited thin film materials. However, less work has focused on obtaining other elastic constants such as Poisson's ratios and shear moduli or the thermal expansion coefficients. In addition, very little account has been taken of the potential for an isotropic material behavior, particularly in the through thickness direction of deposited materials.It is also note worthy that even for a widely use material, such as polysilicon, values of moduli ranging from 132 to $174 \mathrm{GPa}$ have been reported in the literature on material deposited by nominally identical processes. This discrepancy is presumably solely due to differences in experimental technique and illustrates the potential for error associated with obtaining measurements of material properties at the MEMS scale.

\subsection{Strength characterization-}

The characterization of the strength of microfabricated materials is a key issue for MEMS devices which are designed to operate at high mechanical power densities or large deflection levels. The ability to achieve such devices is limited by the strength of the materials of construction. Since the strength of both ductile and brittle materials can be very dependent on the scale and the fabrication route, it is critical that measurements to be used fordesign purposes are obtained from test structures fabricated by the same processing route and at asimilar scale to that to be used for the application for which they are intended. Strength tests on single crystal silicon specimens with surfaces created by different etching "recipes" in the same deep reactive ion etch chamber have been shown to have strengths which can vary by nearly an order of magnitude.Various approaches have been taken to obtain room temperature 
strength related properties. For plastic materials nano-indendation has proven to be a viable means to extract information regarding plastic constitutive behavior. Electrostatic actuation has been used to generate forces su cientto cause fracture in surface micromachined structures. This approach has also been used to derive measurements of the fracture toughness of such materials. However, it has generally been found that in order to generate sufficiently high stresses to cause fracture by such means the cross section of the part has had to be limited to a small fraction of the area used to generate the electrostatic force. In order to test large specimens at higher force levels various studies have used mechanical loading applied via modified micro hardness indentors or nanoindentors to generate bending stresses to cause failure. In addition, tensile tests have been performed using mechanical or electrostatic gripping and in situ strain measurement. These approaches are particularly necessary for the thicker structures realized by bulk micromachining and LIGA processes.Obtaining elevated temperature properties for microfabricated materials is important as the MEMS devices are designed for high temperature applications, as well as to help develop models for microfabrication processes which utilize elevated temperatures for bonding or annealing. Bulge test of pressurized cavities have been used as one means of obtaining such data, as well as more conventional macroscale bend tests and indentation creep tests.

\subsection{Adhesion and bond strength-}

At the heart of virtually all MEMS devices is abasic architecture consisting of multiple layers of materials created by deposition or bonding operations. The structural integrity of the bonds between layers is a key parameter in determining reliability. Several techniques are well established for measuring thin film adhesion including bulge testing, peel testing and residual stress driven cohesion measurements and these are not unique to MEMS devices, although it is worth noting that microfabrication techniques play a key role in creating the test structures which allow these measurements.As previously noted wafer bonding is of more specialized application to MEMS. A number of techniques have been developed to allow determination of bond quality and strength. Non-destructive methods, including infra red, ultrasonic and X-ray imaging, have been employed to detect macroscopic voids. This is particularly valuable during the initial (electrostatic) phase of anodic and fusion bonding operations since poor bonds can be identified and the wafers separated and rebonded before the elevated temperature annealing step is carried out. Bond strength has been characterized by a number of techniques, including pressure burst testing, double cantilever beam specimens and other mechanically loaded structures which expose the bond to combinations of tension and shear stresses. Given the importance of bonding operations to MEMS fabrication this is a fertile area for materials science and mechanical engineering advancement.

\subsection{Residual stresses-}

Since MEMS devices typically contain several deposited and bonded layers of dissimilar materials, residual stresses can play an important role in determining the reliability of the processes and the fabricated devices. The issues of thin film residual stresses have received considerable attention due to their importance in the microelectronics industry, and to a large degree these issues are the same as those found in MEMS. However, as MEMS devices are created which have larger mechanical power and force capabilities, thicker deposited layers are being investigated than are typically utilized in microelectronic applications. This is particularly true in devices that use molding operations, such as LIGA and CVD deposition of SiC.These thicker layers have a greater tendency to fracture and the thickness (and therefore size of thedevice that can be realized) may be limited by the residual stress state.Residual stresses in thin films and other deposited layers arise from several sources: thermal expansion mismatch, incorporation of residual gases 


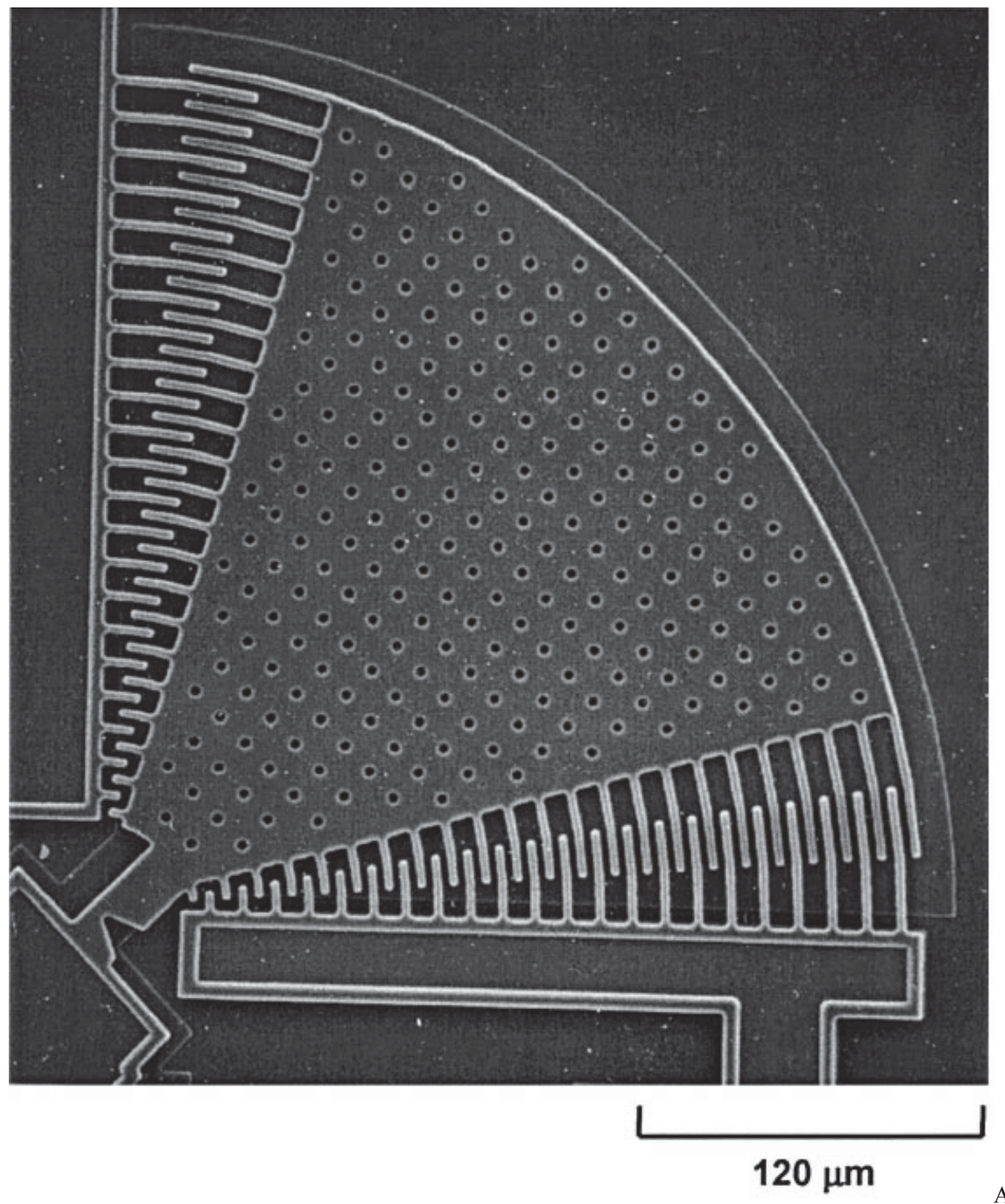

Fig.1.Resonant test structure used to obtain fatigue data for polysilicon (micrograph courtesy of S. Brown, Exponent Failure Analysis Associates).

into deposited materials, lattice mismatch, grain growthand grain size, point defects and sintering. The relative importance of these stress producing mechan-isms depends crucially on the materials, processingconditions and microstructure. The ability to control and characterize residual stresses is very important for the development of higher performance MEMS by MST, and microfabrication techniques offer the possibility of creating novel test structures to permit residual stress characterization.

\subsection{Fatigue-}


Some MST devices may be subject to very high numbers of fatigue cycles during their service lifetimes due to their inherently high operating frequencies. This raises the possibility of fatigue being a limiting factor on the allowable stress levels or useful life. These concerns have resulted in the recent development of test structures to probe the fatigue behavior of microfabricated materials. Typically these structures utilize electrostatic loading and excitation at resonance to obtain streslevels sufficient to cause fatigue failure. Such a structure is shown in Fig. 1. Actuation of the interdigitated electrostatic comb drives allows amoment to be applied to the notched gauge section at the lower left of the figure. Although such test methods are still in development, initial results have shown that fatigue processes can operate in both ductile and brittle microfabricated materials. There is some doubt as to whether the mechanism observed in brittle materials, particularly polycrystalline silicon, is a cyclic fatigue process, or rather an environmentally assisted slowcrack growth process. It is also worth noting that many commercial accelerometers and pressure sensors have experienced extremely high numbers $\left(>10^{\wedge} 8\right)$ of cycles apparently without sustaining any fatigue failures. However, as MEMS devices start to push towards higher mechanical power levels fatigue may increasingly become a concern.

\subsection{Surface forces and tribology-}

The high surface area to volume ratio of MST devices implies that tribological effects are likely to be important factors in determining performance.Experiences with surface micromachined accelerometers and micromotors suggest that surface adhesion due to charge build up or moisture adsorption is a critical issue that results in stiction and hysteresis. The same scaling of electrostatic forces that makes it attractive for prime movers at the microscale also can prove a liability. In addition, the use of a wet etch as the release step for surface micromachined devices can be complicated by the introduction of capillary forces between elements that prevent their separation. Experience with micromotors and micro-gear trains running at high rotational speeds on unlubricated sliding contacts has indicated that wear processes are very important in both allowing the bearing surfaces to be worn in to allow low friction operation, and subsequently in contributing to failure. This is despite the very low inertial and gravitational forces associated with the devices.The importance of tribology for MEMS and in MST has resulted in a growing literature on the subject and quantitative measurements of surface adhesion forces, friction and wear, and erosion behavior have been obtained from a variety of devices. Attempts are being made to modify micromachined surfaces or apply low friction coatings in order to promote better tribological characteristics and there is a great need for increased understanding in this area if reliable and durable devices are to be created. In addition, non materials solutions, involving the use of air bearings or magnetic levitation, offer promise for overcoming some of the tribological issues associated with high speed MEMS and other MST devices.

\section{CONCLUDIG REMARKS}

As we know, MEMS in MST represent a rapidly developing area of technology with great economic potential. Advances in materials science and technology have played key roles in the evolution that has occurred thus far, and will continue to do so in the coming decades. Near term developments are relatively straightforward to forecast in relation to MEMS which are derived from microelectronicdevices and share the microelectronic tool set forfabrication. These areas for advancement include : new material development, fabrication processadvancement and the development of standard mechanical characterization techniques. With regard to new material development the integration of silicon carbide and possibly diamond for mechanical elements and the expansion of the set of possible transducer materials offer great potential for increased performance. With regard to fabrication processes the continued development of masks and etches that can yield high aspect ratio structures and the development of deposition techniques, particularly with regard to creating thicker coatings with reduced residual stress levels, are key activities.

The development of standard characterization techniques, particularly with regard to the mechanical properties, is very important if the full potential for paralleling the simulation-based design methodology achieved for VLSI devices is to be realized.In the longer term, great potential exists for expanding the fabrication tool set for MST, and relaxing the restrictions imposed by closely following processes used for microelectronic fabrication.Particular advances include the development of techniques for creating truly three-dimensional structures, while still allowing for wafer-level "multi-up" fabrication.Advances are being demonstrated in materials chemistry in the creation of self-assembling organicmaterials . These materials offer the promise of radically altering the fabrication tool set and the structures and materials that can be considered as well as permitting consideration of devices at very small scales. It remains to be seen what, if any, role these materials play in MEMS devices as we currently understand the 
term. There is also considerable interest in so-called "Nanotechnology" which presupposes that self assembling devices could be developed at scales several orders of magnitude below those currently occupied by MEMS.

This may be possible, but careful scrutiny needs to be applied since existing MEMS are already at scales where performance is limited by dissipative phenomena such as viscous flow and stiction.Paradoxically the most significant advances in MEMS may occur by developing technologies to produce larger devices with similar unit costs to those for existing microelectronics. These devices would have more useful power and force capabilities than current MEMS and are perhaps more properly termed mesoscale machines.MEMS also offer considerable opportunities to advance the field of materials science at larger scales. Microfabricated probe elements enable atomic force microscopes and scanning tunneling microscopes that have revolutionized surface science and tribology. Microfabricated test structures enable the measurement of properties at small scales for applications other than MEMS. MEMS sensors can also be used as monitors for large scale processes. The development of MEMS devices to monitor microstructural evolution during processing or degradation and damage during operation is also feasible. In addition, microscale chemical and biological reactors may permit the synthesis of novel materials due to the ability to very closely control the conditions under which the synthesis occurs.Key areas for materials science to focus oninclude the extension of the available set of materials that can be microfabricated, the refinement of the set of processes available to microfabricatestructures, and improvement in the methods used to characterize and select materials for MEMS applications. In addressing these issues it is important to do so in the context of MEMS as systems in MST, since materials solutions are only viable if they are compatible with the overall fabrication route and the requirements for the application.

\section{ACKNOWLEDGEMENTS}

The author acknowledges the contributions of many authors in the writing of this article. Inparticular the knowledge gained during coursework at university.I am thankful to Dr.Rakesh kumar for providing me the basic ideas and insights of basic fabrication process of micro and nano structures.

\section{REFERENCES}

[1] Srinivasan, R., Firebaugh, S. L., Hsing, I.-M., Ryley,J., Harold, M. P., Jensen, K. F. and Schmidt, M. A.,’Proceedings, Transducers” '97, 1997 International Conference on Solid-State Sensors and Actuators, Chicago, June 1997, pp. 163-166.

[2] Bisson, C., Campbell, J., Cheadle, R., Chomiak, M.,Lee, J., Miller, C., Milley, C., Pialis, P., Shaw, S.,Weiss, W. and Widrig, C.,"Proceedings, Solid State Sensor and Actuator Workshop", Hilton Head Island,South Carolina, 8-11 June 1998, pp. 1-6.

[3] Bhusan, B. (ed.), "Tribology Issues and Opportunities in MEMS", Proceedings of the NSF/AFOSR/ASME Workshop on Tribology Issues and Opportunities in MEMS, Columbus, OH, 9-11 November 1997.Kluwer, Dordrecht, 1998.

[4] Brown, S., Gilbert, J., Guckel, H., Howe, R.,Johnson, G., Krulevitch, P. and Muhlstein, C. (ed.),"Microelectromechanical Structures for Materials Research", Mater. Res. Soc. Symp. Proc., Vol. 518,1998.

[5] Heuer, A. H. and Jacobs, S. J. (ed.),"Materials Science of Microelectromechanical Systems (MEMS)Devices”, in Mater. Res. Soc. Symp. Proc., Vol. 546,1999.

[6] Spearing,S.M, "Materials issues in microelectromechanical systems(MEMS)", Acta mater. 48 (2000) 179-196

[7] Drexel, K. E., "Nanosystems, Molecular Machines,Manufacturing and Computation". Wiley, New York, 1992.

[8] Ohring, M.,’The Materials Science of Thin Films".Academic Press, London, 1992, pp. 413-438.

[9] Madou, M. J., "Fundamentals of Microfabrication".CRC Press, Boca Raton, FL, 1997.

[10] Ghandhi, S. K. (ed.), "VLSI Fabrication Principles:Silicon and Gallium Arsenide”, 2nd edn. John Wiley,New York, 1994.

[11] Tong, Q. Y., "Semiconductor Wafer Bonding: Science and Technology". John Wiley, New York, 1998.

[12] Ristic, L. J. (ed.), "Sensor Technology and Devices".Artech House, Boston, MA, 1994, Chap. 5/6.

[13] Smith, D. L.,"Thin Film Deposition: Principles and Practice". McGraw-Hill, New York, 1995, pp. 196-197.

[14] Drexel, K. E.,"Nanosystems, Molecular Machines,Manufacturing and Computation. Wiley, New York,1992. 